\title{
Antibiotic prescribing among inpatients with infectious and non-infectious indications in obstetrics and gynaecology departments at two tertiary care hospitals in Central India
}

Anna Machowska

Karolinska Institutet Department of Global Public Health

Kristoffer Landstedt

Karolinska Institutet Department of Global Public Health

Cecilia Stålsby Lundborg

Karolinska Institutet Department of Global Public Health

Megha Sharma ( $\triangle$ megha.sharma@ki.se)

https://orcid.org/0000-0001-9165-9393

\section{Research article}

Keywords: Antibiotic prescribing, Inpatients, Surgical prophylaxis, Bacterial infections, Obstetrics and Gynaecology, Central India

Posted Date: January 30th, 2020

DOI: https://doi.org/10.21203/rs.2.15649/v2

License: (c) (i) This work is licensed under a Creative Commons Attribution 4.0 International License. Read Full License 


\section{Abstract}

Background: Patients in obstetrics and gynaecology (OBGY) departments are at high risk of life-threatening infection, thus prescribed antibiotics extensively. The use of antibiotics leads to increasing antibiotic resistance (ABR). Antibiotic surveillance is one of the cornerstones to combat ABR. However, surveillance of, department specific antibiotic prescription data is rarely performed, especially in low- and middle-income countries. Aim: To describe and compare antibiotic prescription patterns among the inpatients at OBGY departments of two tertiary care hospitals, one teaching (TH) and one non-teaching (NTH), in Central India. Methods: A prospective study was conducted for three years using. Demographics, length of hospital stay, diagnoses and prescribed antibiotics including dose, duration and frequency were recorded for all inpatients during their hospital stay. The patients were divided into, infectious and non-infectious diagnosis categories and further into surgical, non-surgical and possible surgical indications using the International Classification of Disease system version-10. Results: A total of 5558 patients were included in the study; 2044 (81\%) in the TH and 2567 (85\%) in the NTH received antibiotics $(p<0.001)$. A majority of the patients with surgical indications were prescribed antibiotics $(\mathrm{TH}-87 \%, \mathrm{NTH}-100 \%)$. Prescribing of the fixed-dose combinations (FDCs) of antibiotics and use of trade names were more common at the NTH. Most of the inpatients who had neither surgery nor any confirmed bacterial infection received antibiotics (TH$71 \%$ and $\mathrm{NTH}-75 \%)$. Overall, higher DDD/1000 patients were prescribed in the $\mathrm{TH}$ in both categories. Conclusions: High antibiotic prescribing for the inpatients having no infection indications in both hospitals, as well as frequent prescribing of trade-name, broad-spectrum antibiotics, including the FDCs in the NTH than the TH, are points of concern. Investigation of the underlying reasons for prescribing antibiotics for unindicated diagnoses and the development and implementation of antibiotic stewardship programs are recommended measures to improve the antibiotic prescribing practice.

\section{Background}

Antibiotics are broadly used in healthcare facilities, and their use can be quantified by prescription surveillance. The development and implementation of local prescribing guidelines based on the prescription surveillance data and local resistance patterns is a crucial step towards combating antibiotic resistance (ABR). Despite this, the surveillance at healthcare facilities is underperformed, especially in densely populated, low- and middle-income countries (LMICs) like India.

The morbidity and mortality due to the rapid emergence of ABR with subsequent treatment failure of common bacterial infections have increased around the globe (1-3). Use of antibiotics, whether indicated or not, is a significant contributor to the development and spread of $\operatorname{ABR}(2,4-9)$. The global use of antibiotics has increased by $35 \%$ in 2000-2010. An increase of 37\% was observed for antibiotic use in India from 2005 to 2010 (10). The surveillance of the use of antibiotics according to the indications is one of the cornerstones to develop strategies for the improvement of the use of antibiotics and to slow down the development of $\operatorname{ABR}(4,11,12)$. So far, the published studies have not presented the antibiotic prescriptions for specific indications (13-15).

The prescriptions are a reliable and quantifiable source of information to conduct surveillance studies. However, in resource-constrained healthcare settings, patient information, including prescriptions, are generally not computerised and are often documented manually using paper records. This lack of automated data entry makes prescription surveillance an expensive and cumbersome process and is one of the significant contributors to the delay in the development of contextualised antibiotic prescribing guidelines. The lack of local prescribing guidelines obstructs the rational prescribing of antibiotics and might lead to the development of ABR. 
In India, healthcare is provided in both public and private sector healthcare facilities. However, the private sector is a major healthcare provider and constitutes $93 \%$ of all healthcare facilities. Therefore, a major part of antibiotic use in the country can be attributed to private sector hospitals (16-18). Despite this, limited studies have been conducted on the surveillance of antibiotic prescribing at the Indian private healthcare sector, and a few conducted, showed overall high antibiotic prescriptions $(5,13,14)$. These findings are in line with the available evidence of the high use of antibiotics and the presence of various multidrug-resistant bacterial strains in the country $(15,19)$.

Antibiotics are essential in high infection risk departments (where minor and major surgical procedures take place) for both, the treatment of infection and perioperative prophylaxis to prevent healthcare-associated infections (HAls). Although, a majority of the preventable maternal deaths occur in LMICs, including India (20), there is a paucity of studies that assess antibiotic prescribing patterns at the OBGY departments in India.

\section{Methods}

\section{Study setting and design}

This was a prospective, cross-sectional study conducted at the OBGY departments in one teaching hospital (TH) and one non-teaching hospital (NTH) of Ujjain district, Central India. The data was collected for all patients admitted in the OBGY departments between 2008 and 2011. Both study hospitals are private-sector tertiary-care hospitals, run by a not-for-profit charitable trust but differ in mode of providing healthcare services (15).

The TH is situated in a rural area and provides all medical services free of charge. All medicines were purchased by the hospital management and were dispensed free of charge to all patients visiting the $\mathrm{TH}$, during the study. Participation in academic activities is a part of the routine at the TH. The NTH is located in a city area of Ujjain where the patients are charged for the medical consultancy, hospital stay and have to purchase the prescribed medicines out of pocket during their hospital stay (15). The salary structure differs between the hospitals, as in the $\mathrm{TH}$ the physicians/ surgeons receive a fixed salary while the payment of physicians/ surgeons in the NTH is mainly based on the number of patients admitted by them in the hospital.

\section{Data Collection and study population}

The data collection method is described elsewhere in detail $(14,15)$. In brief, the data on antibiotic prescribing was collected manually in the OBGY departments of the two study hospitals. A locally developed and previously used form was used to collect the data on patient's demographics, admission and discharge dates, prescribed antibiotics during the hospital stay and at discharge, (name of antibiotic, dose, duration and frequency). A patient could have more than one diagnosis (indications). All indications, as written in the patients' file at the time of discharge, were noted in the form. The nurses working in the departments were trained to collect the information from the patients' record files in the paper forms. The filled forms were collected from the wards after the patient's discharge.

\section{Inclusion criteria}

Female patients who spent at least one night in the OBGY departments and were more than 15 years were included in the analysis $(21,22)$ (Figure 1$)$.

\section{Data management and analysis}


The diagnoses were grouped according to the International Statistical Classification of Disease and Related Health Problems-Tenth Revision (ICD-10) (23). For some patients the site of the surgery was not specified, or a complaint was recorded as an indication. These indications are not classified by ICD-10 code and, were thus, abbreviated by the authors, such as an abdominal hysterectomy was abbreviated as $\mathrm{AH}$. The diagnoses assigned by the responsible consultant and registered in the patients' file were considered as final and were not validated externally.

Based on the information in patients' records, the patients were divided into, two categories: infectious diagnosis and non-infectious diagnosis. The infectious diagnosis category comprised of all patients with confirmed or suspected infectious diseases including bacterial, viral and fungal infections or having the clinical signs of infection such as fever. The non-infectious diagnosis category comprised of all patients who had no documentation of infectious indication or no clinical signs of any infection. In the next step, both the infectious and non-infectious diagnosis categories were further divided into three diagnosis groups: surgical, non-surgical and possible surgical (Figure 1). Patients who had an indicated or confirmed surgery status were assigned to the surgical patient group. Patients who had no indication for surgery were included in the non-surgical group. Patients that had a diagnosis where a surgical procedure was indicated but the status of the surgery was not specified were included in the possible surgical patient group.

Data analysis was performed anonymously using a unique patient code system assigned after completing the data collection. The prescribed antibiotics were categorised as per the Anatomical Therapeutic Chemical (ATC) classification system according to the WHO Collaborating Centre for Drug Statistics Methodology (WHOCC) (24). In both hospitals, antibiotics could have been prescribed by either generic or trade names. In case an antibiotic was prescribed by trade name, the corresponding generic name was also entered to facilitate the analysis according to the WHO methodology at the substance level of the ATC. The Defined Daily Doses (DDD), as suggested by WHOCC, was used as a unit for the analysis (24).

The local antibiotic prescribing guidelines were not available in any of the study hospitals. The prescribing patterns were compared with the National List of Essential Medicines of India (NLEMI) and the WHO List of Essential Medicines $(25,26)$. Levels of adherence of prescriptions to the NLEMI were analysed and compared between the hospitals (25). The NLEMI is relevant for the national context and thus the recommendations from the NLEMI were followed in the present study (25). For the new FDCs of antibiotics for which ATC codes were not assigned by the WHOCC, the earlier generated codes, J01RA*, were used $(14,15)$.

Data were analysed using Excel, STATA software version 15.0 (Stata Corp., College Station, Texas, USA) and SPSS Statistics version 22 (SPSS Inc, Chicago, IL, USA). For continuous variables sum, mean and standard deviations were calculated. For the categorical variables, frequency and percentage were calculated. Decimals were rounded off to the nearest number. The independent samples t-test was used for comparison of continuous variables since the variables followed a normal distribution. For comparisons of categorical values, Pearson chi-square was used. P-values $\leq 0.001$ were considered significant, according to Bonferroni's correction.

\section{Results}

A total of 6208 patients were admitted in both hospitals and 5558 patients fulfilled the inclusion criteria, that is, 2539 (46\%) patients in the TH and 3019 (54\%) in the NTH (Figure 1). A high proportion of inpatients were prescribed antibiotics in both hospitals. This proportion was higher in the NTH than in the TH $(85 \%$ vs $81 \%$ 
respectively, $\mathrm{p}<0.001)$. Both, the duration of hospital stays, and the duration of antibiotic treatment were significantly longer in the TH (Table 1).

A normal vaginal delivery (FTND, ICD10- 0 80.9, non-surgical group) was the most commonly registered indication at admission in both hospitals, 209/2539 (8\%) in the TH and 572/3019 (19\%) in the NTH. The next common indications in the TH were uterine prolapse $(192,8 \%)$ and vaginal hysterectomy $(\mathrm{VH}, 182,7 \%$, surgical group). At the NTH, surgical procedures such as lower segment caesarean section (LSCS, 480, 16\%) and abdominal hysterectomy $(\mathrm{AH}, 328,11 \%)$ were the next most common indications.

Although a significant difference was not observed between the patients admitted to the TH and NTH, it seems that more ill patients were admitted to the TH. This can be due to the rural catchment area of the TH and more patients from villages visiting the $\mathrm{TH}$. These patients have delayed healthcare seeking behaviour what may explain the reason for the presence of more ill patients at the TH than at the NTH (27).

Figure 1 Patients' categorization based on the recorded indications at the Obstetrics and Gynaecology departments of two private-sector hospitals, a TH and NTH in Central India

Abbreviations: $\mathrm{NTH}=$ non-teaching hospital; OBGY= Obstetrics and gynaecology departments; $\mathrm{TH}=$ teaching hospital

Table 1 Patient's characteristics and antibiotic prescribing at the Obstetrics and Gynaecology departments of two private-sector hospitals, a TH and NTH in Central India

\begin{tabular}{lll}
\hline & TH & NTH \\
\hline Total number of inpatients & 2539 & 3019 \\
Age; mean years (SD) & $34(13)^{*}$ & $30(11)$ \\
Patients prescribed AB; n (\%) & $2044(81)$ & $2567(85) *$ \\
Duration of hospital stay; mean days (SD) & $8(9)^{*}$ & $3(3)$ \\
Duration of AB treatment; mean days (SD) & $8(7)^{*}$ & $5(3)$ \\
Total number of prescription days & 30616 & 12512 \\
\hline
\end{tabular}

Statistically significant values $(\mathrm{p}<0.001)$ are marked with *.

Abbreviations: $\mathrm{AB}=$ antibiotics, $\mathrm{NTH}=$ non-teaching hospital, $\mathrm{OBGY}=$ Obstetrics and gynaecology departments; $\mathrm{SD}=\mathrm{standard}$ deviation, $\mathrm{TH}=$ teaching hospital

\section{The infectious and non-infectious categories}

A total of 5558 inpatients included in the analysis were divided into infectious (TH-230, NTH-175) and noninfectious (TH-2309, NTH-2844) diagnosis categories and were further divided into surgical, non- surgical and possible surgical groups (Figure 1 and Table 2). The number of prescriptions in both infectious and non-infectious diagnosis categories in the TH was higher than in the NTH across all diagnosis groups. The hospital stays and duration of antibiotic treatment for the patients in the non-infectious diagnosis category were significantly longer in 
the TH than in the NTH regardless if the surgery was performed or not. Moreover, at both hospitals, in surgery groups of the infectious and non-infectious diagnosis categories, the number of patients who received antibiotics for one day was less than $5 \%$. The length of antibiotic administration for one day can be a proxy for antibiotic prophylaxis, which is indicated for the surgical procedure (Table 2).

Seventy-six per cent of 405 inpatients in the infectious diagnosis category were in the non-surgical group (TH-161, and NTH-145), and 88\% (269/306) of those were prescribed antibiotics during their hospital stay. In the non-

infectious diagnosis category, 2204 patients were classified in the non-surgical group, (TH-829, and NTH-1375). In this group, more than $70 \%$ of inpatients received antibiotics during their hospital stay. The majority (98\%) of the inpatients in the possible surgery group had non-infectious indications, and $77 \%$ of the patients were prescribed antibiotics in the $\mathrm{TH}$ and $50 \%$ in the NTH.

\section{Antibiotic prescribing patterns in the categories and groups}

In both, the infectious and non-infectious diagnosis categories, across all three groups (surgical, non-surgical and possible surgical), the use of generic names was more common, and adherence to the NLEMI was higher in the TH than in the NTH (Table 3).

In the infectious diagnosis category, nitroimidazole derivatives (23\% and 24\%) and tetracyclines (19\% and 16\%) were the most commonly prescribed in surgical and non-surgical groups at the $\mathrm{TH}$, respectively (Table 3 ). Overall, in this category, other beta-lactams (J01D) and FDCs were prescribed to a greater extent in the NTH than in the TH $(p<0.001)$. In the surgical group of the infectious diagnosis category, the FDCs of third-generation cephalosporins accounted for $53 \%$ of all prescriptions in the NTH while FDCs were not prescribed at all at the TH.

In the non-infectious diagnosis category, third-generation cephalosporins and the FDCs of antibiotics accounted for $58 \%$ of all prescriptions in the surgical group in the NTH whereas aminoglycosides (18\%), imidazole (17\%) and nitroimidazole derivatives (17\%) were the most commonly prescribed in the $\mathrm{TH}$. In the non-surgical group in the $\mathrm{TH}$, extended-spectrum penicillin and imidazole derivatives constituted $52 \%$ of all prescriptions, whereas in the NTH third-generation cephalosporins, and FDCs constituted 57\% (Table 3).

Table 2 Patients' demographic and prescription details at the category and group levels at the Obstetrics and Gynaecology departments of two private-sector hospitals, a TH and NTH in Central India 
Infectious diagnosis category

$\mathrm{N}=405(\mathrm{TH}=230, \mathrm{NTH}=175)$

\begin{tabular}{|c|c|c|c|c|c|c|}
\hline & \multicolumn{2}{|c|}{ Surgical $(n=89)$} & \multicolumn{2}{|c|}{$\begin{array}{l}\text { Non-surgical } \\
(n=306)\end{array}$} & \multirow{2}{*}{$\begin{array}{l}\text { Possible } \\
(\mathrm{n}=10) \\
\mathrm{TH}\end{array}$} & \multirow{2}{*}{$\begin{array}{l}\text { Surgical } \\
\text { NTH }\end{array}$} \\
\hline & $\mathrm{TH}$ & NTH & $\mathrm{TH}$ & NTH & & \\
\hline \multirow[t]{2}{*}{ Total inpatients in each diagnosis group; $\mathrm{n}$} & 62 & 27 & 161 & 145 & 7 & 3 \\
\hline & & & & 126 & & \\
\hline Inpatients prescribed $\mathrm{AB} ; \mathrm{n}(\%)$ & $57(92)$ & $27(100)$ & $143(89)$ & $(87)$ & $6(86)$ & $3(100)$ \\
\hline Duration of hospital stay; mean days (SD) & $11(10)$ & $6(3)$ & $6(5) *$ & $3(3)$ & $13(5)$ & $3(2)$ \\
\hline Duration of $\mathrm{AB}$ treatment; mean days (SD) & $9(7)$ & $6(3)$ & $6(4)^{*}$ & $3(2)$ & $9(3)$ & $4(2)$ \\
\hline \multicolumn{7}{|l|}{$A B$ prescriptions in each diagnosis group; } \\
\hline \multirow[t]{2}{*}{$\mathrm{n}$} & 974 & 186 & 1592 & 479 & 91 & 16 \\
\hline & & & 393 & & & \\
\hline $\mathrm{AB}$ prescriptions by generic name; $\mathrm{n}(\%)$ & $182(19)$ & $17(9)$ & $(25) *$ & $15(3)$ & $2(2)$ & $0(0)$ \\
\hline Types of ABs substances prescribed; $\mathrm{n}$ & 17 & 22 & 18 & 34 & 10 & 3 \\
\hline ABs prescribed using generic name; $\mathrm{n}(\%)$ & $7(41)$ & $2(9)$ & $8(44)$ & $3(9)$ & $1(10)$ & $0(0)$ \\
\hline Prescriptions of $A B s$ found in the NLEMI; & & & 1084 & 271 & & \\
\hline n $(\%)$ & $681(70)$ & $112(60)$ & $(68) *$ & $(57)$ & $54(59)$ & $3(19)$ \\
\hline \multicolumn{7}{|l|}{ Number of inpatients prescribed $A B s$ for } \\
\hline one day & $2(3)$ & $0(0)$ & $4(2)$ & $12(8)$ & $0(0)$ & $0(0)$ \\
\hline
\end{tabular}

Non-infectious diagnosis category

$\mathrm{N}=5153(\mathrm{TH}=2309, \mathrm{NTH}=2844)$

\begin{tabular}{|c|c|c|c|c|c|c|}
\hline & \multicolumn{2}{|c|}{ Surgical $(\mathrm{n}=2506)$} & \multicolumn{2}{|c|}{$\begin{array}{l}\text { Non-surgical } \\
(n=2204)\end{array}$} & \multirow{2}{*}{$\begin{array}{l}\text { Possible } \\
(n=443)\end{array}$} & \multirow{2}{*}{$\begin{array}{l}\text { Surgical } \\
\text { NTH }\end{array}$} \\
\hline & TH & NTH & TH & NTH & & \\
\hline \multirow[t]{2}{*}{ Total inpatients in each diagnosis group; $\mathrm{n}$} & 1183 & 1323 & 829 & 1375 & 297 & 146 \\
\hline & 1030 & 1268 & & 1025 & & \\
\hline Inpatients prescribed $A B ; n(\%)$ & $(87)$ & $(95)^{*}$ & $592(71)$ & (75) & $216(73)$ & $119(82)$ \\
\hline Duration of hospital stay; mean days (SD) & $13(10)^{*}$ & $6(3)$ & $6(6) *$ & $3(3)$ & $10(9) *$ & $4(4)$ \\
\hline Duration of AB treatment; mean days (SD) & $9(8)^{*}$ & $6(3)$ & $6(4) *$ & $3(2)$ & $8(7)^{*}$ & $4(3)$ \\
\hline $\mathrm{AB}$ prescriptions in each diagnosis & 19024 & 7785 & 5165 & 3484 & 3770 & 562 \\
\hline
\end{tabular}


5757

AB prescriptions by generic name; n (\%)

Types of ABs prescribed; $\mathrm{n}$

ABs prescribed using generic name; n (\%)

Prescriptions of ABs found in the NLEMI;

n (\%)

(78)

(30)*

30

12 (43)*

14920

3881

(50)

(82)*

29 (2)

$72(5)$

15 (2)

$111(8)$

$10(3)$

9 (6)

Statistically significant values $(\mathrm{p}<0.001)$ are marked with *. Abbreviations: $A B=$ antibiotics, NLEMI= National list of essential medicines India,

Table 3 Classes and sub-classes of prescribed antibiotics at the category and group level at the Obstetrics and Gynaecology departments of two private-sector hospitals, a TH and NTH in Central India 
Infectious diagnosis category $\mathrm{N}=(\mathrm{TH}-230, \mathrm{NTH}-175)$

\begin{tabular}{|c|c|c|c|c|c|c|}
\hline & \multicolumn{2}{|l|}{ Surgical } & \multicolumn{2}{|c|}{ Non-surgical } & \multicolumn{2}{|c|}{ Possible Surgical } \\
\hline & $T H$ & $N T H$ & $T H$ & $N T H$ & $T H$ & $N T H$ \\
\hline & $n(\%)$ & $n(\%)$ & $n(\%)$ & $n(\%)$ & $n(\%)$ & $n(\%)$ \\
\hline Total prescriptions & 974 & 186 & 1592 & 479 & 91 & 16 \\
\hline Tetracyclines; J01A, J01AA & $189(19)^{*}$ & $1(1)$ & $251(16)^{*}$ & $3(1)$ & $21(23)$ & - \\
\hline Beta-lactam ABs, penicillin; J01C & $83(9)$ & $20(11)$ & $237(15)$ & $69(14)$ & $7(8)$ & $3(19)$ \\
\hline Extended-spectrum penicillin; J01CA & $66(7)$ & - & $202(13)^{*}$ & $13(3)$ & $7(8)$ & - \\
\hline Comb. of penicillin incl. Beta-lactamase AB; J01CR & $17(2)^{*}$ & $20(11)$ & $29(2)^{*}$ & $56(12)$ & - & $3(19)$ \\
\hline Other Beta-lactam; J01D & $100(10)^{*}$ & $67(36)$ & $103(6)^{*}$ & $174(36)$ & $10(11)$ & - \\
\hline 3rd gen. cephalosporins; J01DD & $90(9)^{*}$ & $55(30)$ & $103(6)^{*}$ & $161(34)$ & $10(11)$ & - \\
\hline Aminoglycoside; J01G & $104(11)$ & $17(9)$ & $180(11)^{*}$ & $21(4)$ & - & - \\
\hline Other aminoglycosides; J01GB & $104(11)$ & $17(9)$ & $180(11)^{*}$ & $18(4)$ & - & - \\
\hline Quinolones; J01M, J01MA & $122(13)$ & 27 (15) & $241(15)$ & $48(10)$ & $9(10)$ & - \\
\hline Fixed dose combination of ABs; J01R, J01RA* & - & $43(23)$ & - & $126(26)$ & - & $13(81)$ \\
\hline Other ABs; J01X & $129(13)$ & $9(5)$ & $183(11)^{*}$ & $20(4)$ & $2(2)$ & - \\
\hline Imidazole derivatives; J01XD & $129(13)$ & $9(5)$ & $183(11)^{*}$ & $20(4)$ & $2(2)$ & - \\
\hline Nitroimidazole derivatives; P01A, P01AB & $224(23)^{*}$ & $2(1)$ & $390(24)^{*}$ & $4(1)$ & 30 (33) & - \\
\hline
\end{tabular}

Non -infectious diagnosis category $\mathrm{N}=(\mathrm{TH}-2309, \mathrm{NTH}-2844)$

\begin{tabular}{|c|c|c|c|c|c|c|}
\hline & \multicolumn{2}{|l|}{ Surgical } & \multicolumn{2}{|c|}{ Non-surgical } & \multicolumn{2}{|c|}{ Possible Surgical } \\
\hline & $T H$ & $N T H$ & $T H$ & $N T H$ & $T H$ & $N T H$ \\
\hline & $n(\%)$ & $n(\%)$ & $n(\%)$ & $n(\%)$ & $n(\%)$ & $n(\%)$ \\
\hline Total prescriptions & 19024 & 7785 & 5165 & 3484 & 3770 & 562 \\
\hline Tetracyclines; J01A, J01AA & $1777(9)^{*}$ & $53(1)$ & $158(3)^{*}$ & $25(1)$ & $350(9)^{*}$ & $3(1)$ \\
\hline Beta-lactam ABs, penicillin; J01C & $2791(15)^{*}$ & $606(8)$ & $2156(42)^{*}$ & $543(15)$ & $400(11)$ & $48(9)$ \\
\hline Extended-spectrum penicillin; J01CA & $2466(13)^{*}$ & $61(1)$ & $1858(36)^{*}$ & $296(8)$ & $304(8)^{*}$ & $6(1)$ \\
\hline Comb. of penicillin incl. Beta-lactamase $A B ;$ J01CR & $279(1)^{*}$ & $545(7)$ & $290(6)$ & $247(7)$ & $93(2)^{*}$ & $42(7)$ \\
\hline Other Beta-lactam; J01D & $1316(7)^{*}$ & $2689(35)$ & $390(8)^{*}$ & $1410(40)$ & $478(13)^{*}$ & $182(32)$ \\
\hline 1stgen. cephalosporins; J01DB & $137(1)$ & $57(1)$ & $23(0)^{*}$ & $53(2)$ & $59(2)$ & $3(1)$ \\
\hline 2nd gen. cephalosporins; J01DC & $12(0)^{*}$ & $297(4)$ & - & $151(4)$ & $19(1)^{*}$ & $31(5)$ \\
\hline 3rd gen. cephalosporins; J01DD & $1167(6)^{*}$ & $2335(30)$ & $363(7)^{*}$ & $1193(34)$ & $400(11)^{*}$ & $148(26)$ \\
\hline
\end{tabular}




\begin{tabular}{|c|c|c|c|c|c|c|}
\hline Sulfonamide with trimethoprim; J01E, J01EE & $131(1)^{*}$ & $8(0)$ & $20(0)$ & $10(0)$ & $46(1)$ & - \\
\hline Macrolides, lincosamides J01F & $7(0)^{*}$ & $34(0)$ & $12(0)^{*}$ & $34(1)$ & $18(1)$ & - \\
\hline Macrolides; J01FA & $7(0)^{*}$ & $23(0)$ & $5(0)^{*}$ & $29(1)$ & $9(0)$ & - \\
\hline Aminoglycoside; J01G & $3379(18)^{*}$ & $528(7)$ & $734(14)^{*}$ & $156(4)$ & $694(18)^{*}$ & $58(10)$ \\
\hline Other aminoglycosides; J01GB & $3379(18)^{*}$ & $522(7)$ & $734(14)^{*}$ & $156(4)$ & $694(18)^{*}$ & $58(10)$ \\
\hline Quinolones; J01M, J01MA & $3071(16)$ & $1317(17)$ & $432(8)^{*}$ & $403(11)$ & $544(14)$ & $64(11)$ \\
\hline Fixed dose combination of ABs; J01R, J01RA* & $17(0)^{*}$ & $2217(28)$ & - & $801(23)$ & $2(0)^{*}$ & $159(28)$ \\
\hline Other ABs; J01X & $3364(18)^{*}$ & $300(4)$ & $804(16)^{*}$ & $100(3)$ & $645(17)^{*}$ & $45(8)$ \\
\hline Imidazole derivatives; J01XD & $3320(17)^{*}$ & $300(4)$ & $804(16)^{*}$ & $100(3)$ & $636(17)^{*}$ & $40(7)$ \\
\hline Drugs for treatment of tuberculosis; J04A, J04AM & - & $3(0)$ & - & - & - & $3(1)$ \\
\hline Nitroimidazole derivatives; P01A, P01AB & $3162(17)^{*}$ & $30(0)$ & $459(9)^{*}$ & $10(0)$ & $593(16)$ & - \\
\hline
\end{tabular}

Statistically significant values $(\mathrm{p}<0.001)$ are marked with *. Abbreviations: $A B=$ antibiotics, ATC=The Anatomical Therapeutic Chemical Classification System, $\mathrm{n}=$ number of prescriptions, NTH=non-teaching hospital, OBGY= Obstetrics and Gynaecology departments, TH= teaching hospital

\section{Antibiotic prescribing in DDD/1000 patients}

DDD per 1000 patients was calculated at the substance level based on the ATC methodology. The antibiotics accounting for $90 \%$ of all prescriptions (DU90\%) for each diagnoses group are presented in Table 4 . Overall, higher DDD/1000 patients were prescribed in the TH than in the NTH in both categories. In the TH, regardless of the presence of an infectious indication, doxycycline (in surgical and possible surgical groups) and ampicillin (in the non-surgical group) were the highest prescribed DDD/1000 patients. In the infectious diagnosis category of the NTH, cefixime was most commonly prescribed in the surgical group, ceftriaxone in the non-surgical group and metronidazole in the possible surgical group, whereas in the non-infectious diagnosis category cefixime was most frequently prescribed (Table 4).

Table 4 DDD/1000 patients for the most prescribed antibiotic substances (DU90\%) at category and group level at the Obstetrics and Gynaecology departments of two private-sector hospitals, a TH and NTH in Central India 


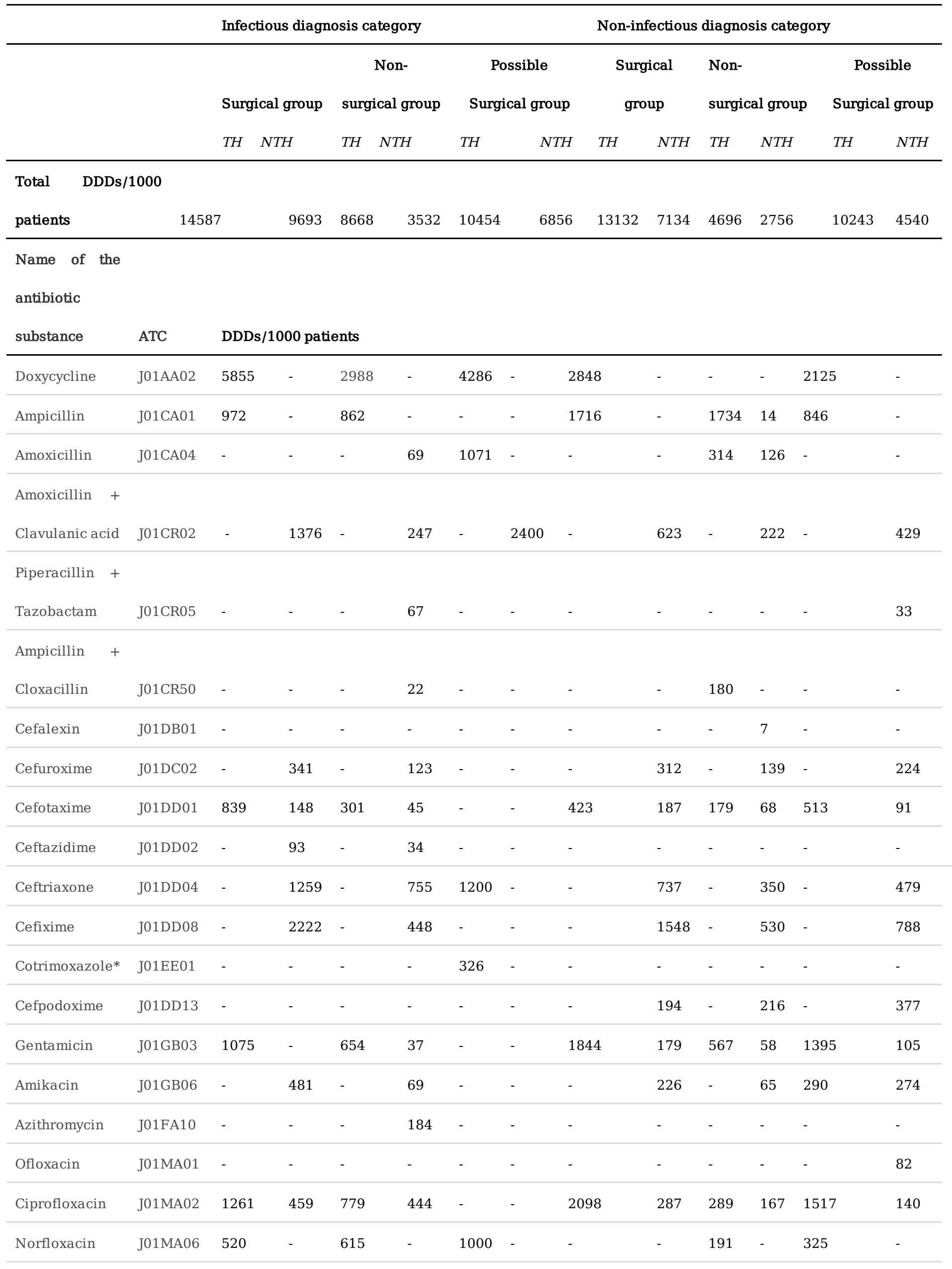




\begin{tabular}{|c|c|c|c|c|c|c|c|c|c|c|c|c|c|}
\hline Levofloxacin & J01MA12 & - & 1111 & - & 131 & - & - & - & 1083 & - & 174 & - & 340 \\
\hline Moxifloxacin & J01MA14 & - & - & - & - & - & - & - & 118 & - & - & - & 60 \\
\hline \multicolumn{14}{|l|}{ Ceftazidime +} \\
\hline Tazobactam & J01RA*82 & - & - & - & - & - & - & - & - & - & - & - & 34 \\
\hline \multicolumn{14}{|l|}{ Ciprofloxacin + } \\
\hline Tinidazole & J01RA*73 & - & - & - & 19 & - & - & - & - & - & - & - & - \\
\hline \multicolumn{14}{|l|}{ Cefoperazone } \\
\hline + Sulbactam & J01RA*83 & - & 259 & - & 30 & - & - & - & 56 & - & 16 & - & 159 \\
\hline \multicolumn{14}{|l|}{ Ceftriaxone +} \\
\hline Sulbactam & J01RA*84 & - & 315 & - & 159 & - & - & - & 177 & - & 63 & - & 253 \\
\hline \multicolumn{14}{|l|}{ Ceftriaxone +} \\
\hline Tazobactam & J01RA*85 & - & 537 & - & 352 & - & 2000 & - & 916 & - & 242 & - & 392 \\
\hline \multicolumn{14}{|l|}{ Cefotaxime $\quad+$} \\
\hline Sulbactam & J01RA*86 & - & - & - & 193 & - & - & - & 278 & - & 120 & - & 41 \\
\hline Cefixime & & & & & & & & & & & & & \\
\hline Ofloxacin & J01RA*91 & - & 833 & - & - & - & - & - & - & - & 127 & - & - \\
\hline \multicolumn{14}{|l|}{ Ofloxacin } \\
\hline Metronidazole & J01RA*72 & - & - & - & - & - & 2456 & - & 82 & - & - & - & 87 \\
\hline \multicolumn{14}{|l|}{ Metronidazole } \\
\hline (parenteral) & J01XD01 & 1960 & 259 & 1088 & 104 & - & - & 2643 & 131 & 911 & 52 & 2066 & 152 \\
\hline \multicolumn{14}{|l|}{ Metronidazole } \\
\hline (oral) & Р01AB01 & 2105 & - & 1381 & - & 2571 & - & 1560 & - & 331 & - & 1166 & - \\
\hline
\end{tabular}

\footnotetext{
* Sulfamethoxazole + trimethoprim, Abbreviations: ATC= Anatomical Therapeutic Chemical Classification code, DDD= Defined Daily Dose, J01RA*= ATC codes according to Sharma et al. (14), NTH= Non-teaching hospital, OBGY= Obstetrics and gynaecology departments, TH= Teaching hospital
}

\section{Discussion}

To the best of our knowledge, this is the first study that describes and compares antibiotic prescription in relation to the presence of infection and surgery conducted among the inpatients at the OBGY departments of two private hospitals in India. In the present study, the patients were categorised based on the presence or absence of 
infectious indications and were further divided into surgical, non-surgical and possible surgical groups. A few studies conducted either at the hospital level or for the shorter period are published $(5,13,14)$. However, none of these studies presents the prescribing patterns in the OBGY department for the stated categories and groups. It is clear, therefore that a comparison of our results with other studies can be done only to a limited extent.

The results of our study indicate several trigger factors for the development of the ABR. Our results showed an overall high antibiotic prescribing rate, as more than $80 \%$ of patients received antibiotics during their hospital stay. This result is comparable to that of a study conducted by Sharma et al. (86\%) (14) but is lower than that of a the study conducted in a rural hospital in Andhra Pradesh, India by Alvarez et al (92\%) (5). In both studies, the analysis was conducted for all admitted patients but indications for prescribed antibiotics were not considered in any of the studies. In the present study, we analysed the data corresponding to the indications at both hospitals and observed extensive antibiotic prescribing to the patients without any record of an infectious indication. We observed that the patients who underwent surgery were prescribed antibiotics to a higher extent (>85\%) than those in the non-surgical group ( $>70 \%)$. However, prescribing antibiotics for the patients who neither underwent surgery nor had any signs of infection cannot be explained at present and need a further detailed study.

\section{Adherence to guidelines}

The prescriptions in the TH adhered more to the NLEMI than those in the NTH and were more frequently made using generic names. A similar pattern was observed in the infectious diagnosis category in both hospitals. One of the possible explanations for the observed trade name prescribing patterns in the NTH is that the consultants associated with the NTH can run private practices, where they are free to meet the medical representatives of pharmaceutical companies. These meetings may often influence the content of the consultant's prescriptions. Studies show that the medical representatives lure consultants into prescribing antibiotics by the trade name, favouring the sale of the products of a particular pharmaceutical company (28). On the other hand, at the TH as per the hospital policy, the consultants are not allowed to run a private practice, and their interaction with medical representatives is restricted. Also, the hospital management prefers to purchase generic medicines over the branded medicines, and supply those to the hospital pharmacy. In addition, a positive impact of continuing medical education seminars held at the TH as a part of the routine academic activity, might also have motivated consultants towards adhering to the recommendations. These hospital policies could be modified contextually and implemented at other similar settings to rationalise antibiotic prescriptions.

\section{Hospitalisation and antibiotic treatment duration and treatment costs}

Patients admitted to the TH had both; significantly longer hospital stays and durations of antibiotic treatment, compared to the inpatients in the NTH (Table 1). These long durations of stay and antibiotic prescription days are also the risk factors for HAls. Suspicion of the presence of HAls or their actual presence results in antibiotic prescriptions (29). Data on the prevalence of HAls was not available in the study hospitals for the entire study duration, due to the absence of computerised records and reduced utilisation of diagnostic facilities. Therefore, it will be inappropriate to comment on the relationship between the HAls and antibiotic prescribing. However, the HAI prevalence could be expected to be similar to those in other comparable settings (30).

In-hospital days are directly related to receiving more prescriptions and increased treatment costs. At the NTH, the healthcare services were subsidised but at the $\mathrm{TH}$ the services were free of charge thus, the patients might have opted to get an early discharge from the NTH mainly when they paid out-of-pocket (15). 


\section{Antibiotic prescribing in infectious and non-infectious categories}

Choice of the drugs both at the category and group levels varied significantly between the hospitals. The FDCs (J01RA*) and third generation cephalosporins were frequently prescribed at the NTH, constituting more than half of the prescriptions. These FDCs are neither included in the WHO list of essential medicines nor the NLEMI (14). Combining antibiotic substances to prepare an FDC is often considered as irrational and costlier $(3,14,31)$. The prescriptions of broad-spectrum antibiotics and FDCs results in unnecessary exposure to antibiotics and increases the risk of $A B R$. This issue needs an immediate attention of the policymakers and the prescribers $(14,15)$.

The presence of bacterial infection is an appropriate indication for antibiotic treatment in post-operative procedures, whereas a surgery per se is an indication to receive a single prophylactic dose of antibiotic before or during the surgery (32). In our study, contrary to the recommendations, antibiotics were prescribed even in the absence of any clinical decision or laboratory confirmation of the presence of an infection. More than $70 \%$ of patients in the non-infectious diagnosis category and non-surgical group were also prescribed antibiotics in both hospitals (Table 2). These results are comparable with a study at an Indian hospital where nearly $70 \%$ of women with severe pre-eclampsia or eclampsia, which is not an indication for antibiotic use, were prescribed antibiotics (33). Most of these women had LSCS (54\%). Prescribing prophylactic antibiotics for surgeries such as LSCS is recommended while in our study, only $23 \%$ of patients had LSCS (33). Prescribing antibiotic treatment for the noninfectious, non-surgical cases is unindicated and increases the risk of ABR. The underlying factors affecting the prescribing patterns at the settings demand a focused study.

\section{Antibiotic prescribing in the groups}

Our results from the surgical groups showed that a large proportion of the inpatients, who did not have a confirmed infection, received antibiotic prescriptions. Moreover, the duration of prescribing antibiotics exceeded the recommended prophylactic duration among almost all inpatients in the surgery groups of both categories $(34,35)$. Prescribing antibiotics as treatment is indicated only in cases of a postoperative or simultaneous infection for procedures such as caesarean sections and hysterectomies (35). Comparable prescription patterns have previously been presented in two Indian studies; by Sharma et al. and Kumar et al., where patients were treated with antibiotics for multiple days after caesarean section instead of single-dose prophylaxis $(33,36)$. Prescribing of antibiotics for extended durations to non-surgical patients increases not only the risk of the developing ABR but also the treatment cost.

A survey conducted among 650 surgeons in India reflected that surgeons do prescribe antibiotics for an more extended periods than recommended in the standard surgeon's guidelines and recommendations (37). A lack of local prescription guidelines could be one of the underlying reasons for the observed high antibiotic prescriptions. Interventions such as the development and successful implementation of relevant antibiotic prophylaxis guidelines and regular prescription audits would help to reduce antibiotic prescription, as demonstrated for LSCS patients in a single centre study from Serbia (38). This study showed a significant post-intervention reduction in the use of third generation cephalosporins and of the total treatment cost by $47 \%$ (38). The precise underlying reasons for prescribing antibiotics for extended durations and for unindicated conditions in the present study settings are not clear. Therefore, we recommend further investigation using a qualitative approach among the prescribers at both hospitals.

In the non-surgical groups at both hospitals, FTND was the most common condition for admission and a majority of the patients who gave birth to FTND also received antibiotic treatment. Antibiotic prescription to this group of 
patients cannot be justified as even the prophylactic use of antibiotics for FTND is not recommended (39). A previous study from Ujjain district in India has shown an antibiotic prescribing to 87\% FTND patients (40).An episiotomy is a possible indication for antibiotic prophylaxis in FTND; however, according to a Cochrane review, further studies are required to ascertain the need for antibiotic prophylaxis for an episiotomy (41). Thus, a targeted study is suggested to investigate the rationality of prescribing antibiotics for FTND.

\section{Future implications}

- Feedback to the prescribers on the current situation in OBGY departments

- Qualitative approach to explain the underlying reason for antibiotic prescribing for non-indicated conditions and for a longer time than recommended

- Emphasis on infection prevention through motivating hygiene in hospital and community

- Continue prescription follow up and audits

\section{Strengths and limitations}

The major strength of this study is the relatively large population of 5558 patients included in the analysis. The data was collected over three years, which facilitated to study the antibiotics prescribing patterns for an extended period.

Additionally, the data was collected comprehensively for every inpatient to overcome the selection bias. All diagnoses were checked manually from the patients' files and patients were divided into the diagnosis groups in consultation with two local obstetricians and gynaecologists for comprehensive categorisation. The consultants were not identified at any stage of the study. This method of data collection might have given consultants the freedom to decide on the treatment plan for the patients.

However, this study must be seen in the context of its limitations. First, since the data was collected manually, the possibility of missing data was foreseen. For the missing data, the records in the archive were checked before the analysis. For a small proportion of patients, information on whether the patients were operated on or not, could not be retrieved. For such patient records, a third diagnosis group, "possible surgical" was created to nullify the probable overestimation of antibiotic prescribing. Secondly, the diagnoses (indications) were not validated externally. Also, the use of DDD has been criticised because it has shown a weak correlation with prescribed daily doses in some settings. However, prescribed daily doses may vary among healthcare facilities, and DDD allows comparison among hospitals or clinics even when prescribed daily doses are different. Finally, the use of personal identification numbers, the presence of inexperienced staff for data collection, high staff turnover and the absence of computerised record systems in hospitals make a comprehensive study like this time-consuming and tedious, which causes a delay in analysis. We are aware that extensive manual checking and adding of the ICD codes, and ATC codes for the new FDCs in the data have prolonged the analysis and delayed the presentation. However, the use of human resources is the only way to conduct such detailed studies at resource-constrained settings leads to a more accurate description of the prescribing patterns. Although, the data represented in this paper covers the time from 2008 to 2011, a similar pattern of antibiotic use has been predicted through an extrapolation model by Tamhankar et al. Thus, it indicates that the patterns presented in our study is similar to the one in recent years. This increases the validity of the results of our study in the present context (42).

\section{Conclusions}

Page $15 / 20$ 
High antibiotic prescribing was observed in OBGY departments in both hospitals; however, it was more common in the NTH than in the TH. Antibiotics were prescribed empirically to the patients without any clinical indications of infection in both hospitals. Patients that underwent elective surgeries received courses of antibiotics lasting several days, despite the recommendations to prescribe a single-dose prophylaxis. Broad-spectrum antibiotics, including the new FDCs, were more frequently prescribed by trade names at the NTH than the TH.

A multiple-step approach including an antibiotic stewardship program, is suggested to address the issue of overprescribing of antibiotics. The program could include; the presentation of the study results as feedback to the consultants, the involvement of the consultants in the development of local diagnosis-specific antibiotic prescribing guidelines, the implementation of the guidelines and, periodic training and educational sessions. The antibiotic stewardship needs to be accompanied by continuous surveillance of the prescriptions and qualitative studies to explore the underlying reasons for the present prescription patterns.

\section{List Of Abbreviations}

$\mathrm{AH}-$ Abdominal hysterectomy

ATC- Anatomical Therapeutic Chemical

DDD- Defined Daily Doses

FDCs- Fixed-dose combinations

FTND- Full-term normal delivery

HAls- Healthcare-associated infections

ICD 10- International Statistical Classification of Diseases and Related Health Problems- Tenth Revision

LCSC- Lower segment caesarian section

LMICs- Low and middle- income countries

NLEMI- National List of Essential Medicines of India

NTH- Non-teaching hospital

OBGY- Obstetrics and Gynecology

TH-Teaching hospital

WHO- World Health Organization

WHOCC- WHO Collaborating Centre for Drug Statistics Methodology

\section{Declarations}

Ethics approval and consent to participate 
The ethics committee of Ruxmaniben Deepchand Gardi Medical College, Ujjain, approved the study with the number: $41 / 2007$ and 114/2010. This was an observational study where the data collection was done using patient files. The study did not interfere with the patients receiving the treatment, and none of the patients was contacted during the study period. Each patient was given a unique code. The analysis was performed using these unique codes to maintain confidentiality. No exclusions were made by age, sex or other demographic criteria.

\section{Consent for publication}

Not applicable

\section{Availability of data and material}

The data are available to all interested researchers upon request made to; The Chairman, Ethics Committee, R. D. Gardi Medical College, Agar Road, Ujjain, Madhya Pradesh, India 456006 (Email:

iecrdgmc@yahoo.in, uctharc@bsnl.in),giving all details of the article. The ethical approval number: 41/ 2007 and $114 / 2010$ to be quoted along with the request.

\section{Competing interests}

The authors have no competing interests to declare.

Funding Swedish Research Council (K2007-70X-20514-01-3, K2010-

396 70X-20514-04-3, 2017-01327) and Asia Link (348-2006-6633). MS is the recipient of Erasmus Mundus Lot-15.

\section{Authors' contributions}

MS and CSL designed and initiated the study. AM, MS, analysed the data. AM, MS, and KL interpreted and presented the data and were responsible for drafting the manuscript. CSL, KL and MS gave critical suggestions for the manuscript. All authors read and approved the final manuscript.

\section{Acknowledgements}

The authors extend thanks to the nursing staff of both hospitals involved in the data collection, to the management of both hospitals, to the consultants of the OBGY departments and Dr V.K. Mahadik (Medical Director) for the permission and support during the study.

\section{References}

1. J. ON. Antimicrobial resistance: tackling a crisis for the health and wealth of nations, The review on antimicrobial resistance. 2014.

2. Kotwani A, Holloway K. Access to antibiotics in New Delhi, India: implications for antibiotic policy. J Pharm Policy Pract. 2013;6:6.

3. Organization WH. The evolving threat of antimicrobial resistance: options for action: World Health Organization; 2012.

4. Westly E. India moves to tackle antibiotic resistance. Nature. 2012;489(7415):192. 
5. Alvarez-Uria G, Zachariah S, Thomas D. High prescription of antimicrobials in a rural district hospital in India. Pharm Pract (Granada). 2014;12(2):384.

6. Morgan DJ, Okeke IN, Laxminarayan R, Perencevich EN, Weisenberg S. Non-prescription antimicrobial use worldwide: a systematic review. Lancet Infect Dis. 2011;11(9):692-701.

7. Laxminarayan R, Heymann DL. Challenges of drug resistance in the developing world. BMJ. 2012;344:e1567.

8. Bell BG, Schellevis F, Stobberingh E, Goossens H, Pringle M. A systematic review and meta-analysis of the effects of antibiotic consumption on antibiotic resistance. BMC Infect Dis. 2014;14:13.

9. Bronzwaer SL, Cars O, Buchholz U, Molstad S, Goettsch W, Veldhuijzen IK, et al. A European study on the relationship between antimicrobial use and antimicrobial resistance. Emerg Infect Dis. 2002;8(3):278-82.

10. Van Boeckel TP, Gandra S, Ashok A, Caudron Q, Grenfell BT, Levin SA, et al. Global antibiotic consumption 2000 to 2010: an analysis of national pharmaceutical sales data. Lancet Infect Dis. 2014;14(8):742-50.

11. Okeke IN, Laxminarayan R, Bhutta ZA, Duse AG, Jenkins P, O'Brien TF, et al. Antimicrobial resistance in developing countries. Part I: recent trends and current status. Lancet Infect Dis. 2005;5(8):481-93.

12. Organization WH. Global Action Plan on Antimicrobial Resistance. 2015.

13. Kumar R, Indira K, Rizvi A, Rizvi T, Jeyaseelan L. Antibiotic prescribing practices in primary and secondary health care facilities in Uttar Pradesh, India. J Clin Pharm Ther. 2008;33(6):625-34.

14. Sharma M, Eriksson B, Marrone G, Dhaneria S, Lundborg CS. Antibiotic prescribing in two private sector hospitals; one teaching and one non-teaching: a cross-sectional study in Ujjain, India. BMC Infect Dis. 2012;12:155.

15. Sharma M, Damlin AL, Sharma A, Stalsby Lundborg C. Antibiotic prescribing in medical intensive care units-a comparison between two private sector hospitals in Central India. Infect Dis (Lond). 2015;47(5):302-9.

16. Deshpande K, RaviShankar, Diwan V, Lonnroth K, Mahadik VK, Chandorkar RK. Spatial pattern of private health care provision in Ujjain, India: a provider survey processed and analysed with a Geographical Information System. Health Policy. 2004;68(2):211-22.

17. Organization WH. Health statistics 2009 [Available from: http://who.int/whosis/whostat/EN_WHS09_Full.pdf.

18. P C. Healthcare in India: Features of one of the most privatised systems in the world; 1 September 2009.

19. Kumar SG, Adithan C, Harish BN, Sujatha S, Roy G, Malini A. Antimicrobial resistance in India: A review. J Nat Sci Biol Med. 2013;4(2):286-91.

20. Organization WH. World Health Statistics. 2014.

21. John J. IAP Policy on Age of Children for Pediatric Care. Indian Pedciatrics. 1999;36:461-3.

22. Organization WH. Introduction to Drug Utilization Research. 2003.

23. Organization WH. International Statistical Classificatoion of Disease and Related Health Problems - 10th revision. 2010.

24. Organization WH. Collaborating centre for drug statistics methodology, anatomical therapeutic chemical classification with defined daily doses 2017 [Available from: https://www.whocc.no/atc_ddd_index/.

25. Directorate General of Health Services MoHaFW, Government of India. National List of Essential Medicines 2011 [Available from: https://www.nhp.gov.in/national-list-of-essential-medicines-2011_pg.

26. Organization WH. Model Lists of Essential Medicines 2017 [Available from: http://www.who.int/medicines/publications/essentialmedicines/en/. 
27. Khare S, Purohit M, Sharma M, Tamhankar AJ, Lundborg CS, Diwan V, et al. Antibiotic Prescribing by Informal Healthcare Providers for Common Illnesses: A Repeated Cross-Sectional Study in Rural India. Antibiotics (Basel). 2019;8(3).

28. Blumenthal D. Doctors and drug companies. N Engl J Med. 2004;351(18):1885-90.

29. Saviteer SM, Samsa GP, Rutala WA. Nosocomial infections in the elderly. Increased risk per hospital day. Am J Med. 1988;84(4):661-6.

30. Pathak A, Saliba EA, Sharma S, Mahadik VK, Shah H, Lundborg CS. Incidence and factors associated with surgical site infections in a teaching hospital in Ujjain, India. Am J Infect Control. 2014;42(1):e11-5.

31. Organization WH. Global Strategy for Containment of Antimicrobial Resistance. 2001.

32. Crader MF, Bhimji SS. Preoperative Antibiotic Prophylaxis. StatPearls. Treasure Island (FL)2018.

33. Kumar S, Bansal D, Hota D, Jain M, Singh P, Pandey BL. Assessment of clinical outcomes and prescribing behavior among inpatients with severe preeclampsia and eclampsia: an Indian experience. Indian J Pharmacol. 2014;46(1):18-23.

34. Smaill FM, Grivell RM. Antibiotic prophylaxis versus no prophylaxis for preventing infection after cesarean section. Cochrane Database Syst Rev. 2014(10):CD007482.

35. van Schalkwyk J, Van Eyk N, Society of O, Gynaecologists of Canada Infectious Diseases C. Antibiotic prophylaxis in obstetric procedures. J Obstet Gynaecol Can. 2010;32(9):878-92.

36. Sharma JB, Gupta N, Aggarwal P, Mittal S. A survey of obstetricians' practice of using prophylactic antibiotics in vaginal deliveries and caesarean sections. J Indian Med Assoc. 2008;106(3):147-9.

37. Kulkarni Ra KR, Kochhar P, Dargude V, Rajadhyakshya S, et al. . Patterns of antimicrobial use by surgeons in India. Indian J Surg 2005;67(6):308-15.

38. Ristic S, Miljkovic B, Vezmar S, Stanojevic D. Are local clinical guidelines useful in promoting rational use of antibiotic prophylaxis in caesarean delivery? Pharm World Sci. 2010;32(2):139-45.

39. Liabsuetrakul T, Lumbiganon P, Mori R, Gulmezoglu M, Souza JP, Maternal WHOGSo, et al. A secondary analysis of the WHO Global Survey on Maternal and Perinatal Health for antibiotics used in vaginal deliveries. Int J Gynaecol Obstet. 2014;124(3):240-3.

40. Sharma M, Sanneving L, Mahadik K, Santacatterina M, Dhaneria S, Stålsby Lundborg C. Antibiotic prescribing in women during and after delivery in a non-teaching, tertiary care hospital in Ujjain, India: a prospective crosssectional study. J Pharm Policy Pract. 2013;6:9.

41. Liabsuetrakul T, Choobun T, Peeyananjarassri K, Islam M. Antibiotic prophylaxis for operative vaginal delivery. Cochrane Database Syst Rev. 2004(3):CD004455.

42. Tamhankar AJ, Karnik SS, Stalsby Lundborg C. Determinants of Antibiotic Consumption - Development of a Model using Partial Least Squares Regression based on Data from India. Sci Rep. 2018;8(1):6421.

\section{Figures}




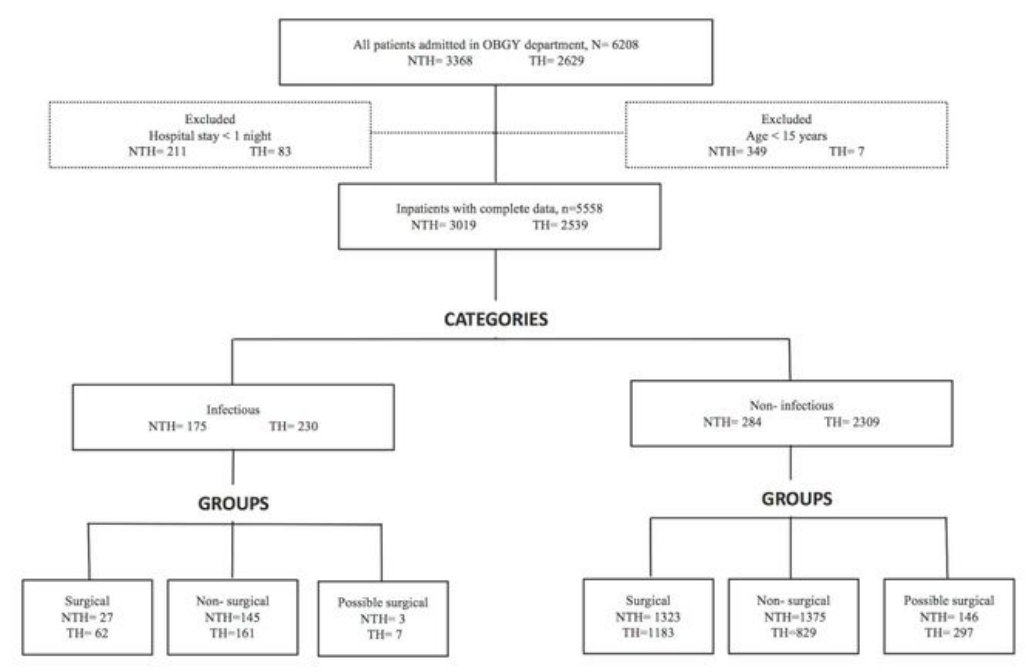

\section{Figure 1}

Grouping of the inpatients based on recorded indications at the Obstetrics and Gynaecology Departments of two private a TH and NTH hospitals in Central India Abbreviations: NTH= non-teaching hospital; OBGY= Obstetrics and gynaecology departments; $\mathrm{TH}=$ teaching hospital 